\title{
Association Between Falls and Chronic Health Conditions Among Adults in Residential Care Facilities: Analysis of the National Survey of Residential Care Facilities (NSRCF) Data
}

\author{
Abeer Alshahrani ${ }^{1 \#}$, Huan-ju Shih ${ }^{1 \#}$, Sean Kim ${ }^{2 \#}$ and Jongwha Chang ${ }^{3 *}$ \\ ${ }^{1}$ College of Health and Human Services, George Mason University, Fairfax, VA, USA \\ ${ }^{2}$ Department of Biopharmaceutical Science, Shenandoah University, Winchester, VA, USA \\ ${ }^{3}$ Department of Pharmaceutical Sciences, The University of Texas, El Paso, TX USA \\ ${ }^{*}$ All three authors contributed equally to this manuscript
}

*Corresponding author: Jongwha Chang, PhD, Department of Pharmaceutical Sciences, School of Pharmacy, University of Texas at

El Paso, 500 W. University Ave, El Paso, TX 79968

ARTICLE INFO
Received: 画 September 25, 2020

Published: 幽 October 01, 2020

Citation: Abeer A, Huna-ju S, Sean K Jongwha C. Association Between Falls and Chronic Health Conditions Among Adults in Residential Care Facilities: Analysis of the National Survey of Residential Care Facilities (NSRCF) Data. Biomed J Sci \& Tech Res 30(5)-2020. BJSTR. MS.ID.005017.

\section{ABSTRACT}

Objective: This study attempts to examine the effects of stroke, heart disease, Alzheimer/dementia, Chronic Obstructive Pulmonary Disease (COPD), age, gender, and Medicaid status on falls for adults in residential care facilities.

Methods: Univariate analysis was conducted to examine the distribution of resident demographic characteristics, health conditions, and Medicaid status for the entire sample. Bivariate analyses (Chi-square test of independence) were also conducted to determine if there were any statistically significant associations between independent and dependent variables. Finally, multivariable logistic regression analyses were conducted to determine the effects of stroke, heart disease, Alzheimer/dementia, and COPD, age, gender, and Medicaid status on the likelihood of any falls in the past year among adult residents in residential care facilities.

Results: Overall, our model is statistically significant compared to an empty model. The multivariable logistic regression model was statistically significant $(\chi 2(8)=116.57, p$ $<0.001)$. Of the seven-predictor variables, only five variables were statistically significant: age, gender, heart disease, Alzheimer's /Dementia, and Medicaid status. COPD and stroke were a not-statistically significant predictor of falls.

Conclusion: Falls have been associated with several different risk factors. Age or gender are risk factors that cannot be altered. However, many other fall risk factors can be controlled (e.g., muscle strength, number of medications, balance). Appropriate assessments can help to identify those residents who have an increased risk of falls. Assessment should include the underlying causes and plans to reduce the negative impact of falls in older adults.

\section{Introduction}

In the United States, falls are the leading cause of both fatal and non-fatal injuries among adults aged 65 and older [1]. Falls can affect one in three adults over the age of 65 and older annually [2]. The costs associated with fall-related injuries present a major burden on our health care system [3]. As many as $20 \%$ of falls can result in serious injuries that may require extensive medical care, including hospitalization and rehabilitation [3]. Hospitalizations of older adults can be associated with adverse outcomes, such as 
functional decline and falls as well as quality of life [4]. In addition, when elderly transition to long-term care $30 \%$ of the elderlies report they are more likely to reduce physical activity [5]. Due to the fact that most residential care facilities do not have the ability to hire sufficient care-givers that allows to assist each resident enough physical exercise, more than $80 \%$ of the time was spent sitting or lying down [6]. Fall rates can vary from 3 to 20 per 1000 bed-days among hospitalized patients [2]. For instance, the association between elderly with other chronic health conditions such as antidepressants with falls, and other risk of falls factors varies from each individual [7]. Another common chronic health issue among elderly is progression of dementia, more than a quarter of older people will have dementia or related symptoms and they are eightfold higher to be at risk of falling compared to those without dementia [8]. Reducing fall rates for adults in residential care facilities has been a priority among healthcare lawmakers due to associated risks related to prolonged hospitalization. Moreover other studies have shown that those who transition to nursing homes have approximate $20 \%$ to $24 \%$ fatal falls with approximately 1.5 falls occurring per nursing home bed per year $[9,10]$.

The statistical results have provided large amounts of evidence that there are more fall risk tools that need to be addressed. A large and growing body of literature has identified risk factors that can increase the incidence of hospitalizations among residents with congestive heart failure (CHF), circulatory or respiratory problems adjusting for age and gender [4]. Although many studies have examined hospitalizations among older adults (aged 65 years and older), fewer studies have investigated the relationship between falls and hospitalizations risk factors among younger and older residents in assisted living and other residential care facilities. The purpose of this study was to examine the prevalence of falls in younger and older adults in residential care facilities from the 2010 National Survey of Residential Care Facilities (NSRCF) data. This study attempts to examine the effects of stroke, heart disease, Alzheimer/dementia, Chronic Obstructive Pulmonary Disease (COPD), age, gender, and Medicaid status on falls for adults in residential care facilities.

\section{Methods}

\section{Study Design \& Data Source}

In 2010, the National Center for Health Statistics collected data on U.S. residents living in residential care settings by conducting proxy interviews with facility administrators. This study utilized a cross-sectional study design from the 2010 National Survey of Residential Care Facilities (NSRCF). Data were collected via inperson interviews with the facility directors or designated staff and not directly with the residents. Data collected on residents included residents' demographics, living arrangements, activities, health conditions (both physical and mental), cognitive and physical functioning, as well as services received. Detailed information about the design and data collection procedures can be found elsewhere
[5]. The sample included around 8,094 residents.

\section{Measures}

The variable selection of the independent variables based on previous literature about risk factors for falls, then we grouped the variables into these categories: demographic characteristics, chronic health conditions, and Medicaid coverage for residents $[2,11,12,13]$. Independent variables for the analysis included demographic characteristics such as age $(\leq 64,65-84$, and $>85)$, and gender (male and female). Other independent variables included chronic health conditions (presence or absence of stroke, heart disease, Alzheimer/dementia, and COPD) and Medicaid coverage for residents. Chronic health condition assessments were done by asking facility administrators the following question: "As far as you know, has a doctor or other health professional ever diagnosed the resident with any of the following conditions?" Our key outcome variable was whether residents experienced falls in the past 12 months based on two survey questions that result from either hip fracture or other injury.

\section{Statistical Analyses}

Univariate analysis was conducted to examine the distribution of resident demographic characteristics, health conditions, and Medicaid status for the entire sample. Bivariate analyses (Chisquare test of independence) were also conducted to determine if there were any statistically significant associations between independent and dependent variables. Finally, multivariable logistic regression analyses were conducted to determine the effects of stroke, heart disease, Alzheimer/dementia, and COPD, age, gender, and Medicaid status on the likelihood of any falls in the past year among adult residents in residential care facilities. Odds ratios and 95\% confidence intervals were computed using STATA, version 13 (Stata Corporation, College Station, TX). For all inferential statistics, an alpha value of 0.05 was used.

\section{Results}

\section{Univariate Analysis}

Table 1 shows the sample size of 7,903 residents at a residential care facility. The majority (over 85\%) of residents were aged above 65 years old. There are 1,200 (15.18\%) residents below or equal to 64 years old; 2,754 (34.85\%) were aged between 65 to 84 , and 3,949 (49.97\%) were aged below 85 years old. In this study, the majority of the population were female with 5,387 (68.16\%) residents compare to 2,516 (31.84\%) male residents. Among the total sample size, there were 1,096 (13.87\%) falls, 867 (10.97\%) residents experienced a stroke, and 3,379 (42.76\%) residents had Alzheimer's or other types of dementia. In addition, 855 (10.82\%) residents from the total sample size had Chronic Obstructive Pulmonary Disease (COPD) while 1,876 (23.74\%) residents had Medicaid coverage. However, the majority of the residents (76.26\%) or 6,027 residents did not have Medicaid coverage. 
Table 1: Distribution of Sample Characteristics.

\begin{tabular}{|c|c|c|}
\hline Variables & $\mathbf{n}$ & Percent( \%) \\
\hline Falls & 7,903 & 100 \\
\hline Yes & 1,096 & 13.87 \\
\hline No & 6,807 & 86.13 \\
\hline Age & 7,903 & 100 \\
\hline$\leq 64$ & 1200 & 15.18 \\
\hline $65-84$ & 2,754 & 34.85 \\
\hline$>85$ & 3,949 & 49.97 \\
\hline Gender & 7,903 & 100 \\
\hline Male & 2,516 & 31.84 \\
\hline Female & 5,387 & 68.16 \\
\hline Stroke & 7,903 & 100 \\
\hline Yes & 867 & 10.97 \\
\hline No & 7,036 & 89.03 \\
\hline $\begin{array}{l}\text { Alzheimer"s/other } \\
\text { Dementia }\end{array}$ & 7,903 & 100 \\
\hline Yes & 3,379 & 42.76 \\
\hline No & 4,524 & 57.24 \\
\hline COPD & 7,903 & 100 \\
\hline Yes & 855 & 10.82 \\
\hline No & 7,048 & 89.18 \\
\hline Paid by medicaid & 7,903 & 100 \\
\hline Yes & 1,876 & 23.74 \\
\hline No & 6,027 & 76.26 \\
\hline
\end{tabular}

\section{Bivariate Analyses}

Table 2 shows the association between falls versus resident characteristics and falls versus chronic medical conditions. There was a statistically significant association between falls and age (categorical data with three levels) for residents' age less than or equal to 64 years old, age between 65 to 84 years old, and greater than equal to 85 years old with a $p$-value $<0.001$. Residents aged greater than or equal to 85 years old were more likely to experience falls (58.49\%) compared to younger aged residents. There was a statistically significant association between falls and gender ( $p$-value $<0.001)$ as female residents were more likely to experience falls (76.9\%) compared to male residents $(23.08 \%)$. Thirdly, there was a statistically significant association between falls and Alzheimer's disease or dementia with a $p$-value $<0.001$. Residents with Alzheimer's disease were more likely to experience falls (51.09\%) compared to those without Alzheimer's diseases or dementia (48.91\%). Lastly, there was a statistically significant association between falls and heart disease ( $p$-value $=0.001)$. Residents with heart disease were more likely to experience falls $(37 \%)$ relative to those without heart disease (63\%). However, there was no significant association between falls vs Medicaid coverage ( $p$-value $=0.584)$, falls vs stroke $(p$-value $=0.898)$, and falls vs COPD ( $p$-value $=0.559)$.
Table 2: Associations between Falls and Resident Characteristics, Medical Conditions, and Medicaid status.

\begin{tabular}{|c|c|c|c|c|}
\hline Variables & Falls & No Falls & & \\
\hline & $\mathrm{n}(\%)$ & $\mathrm{n}(\%)$ & $\begin{array}{c}\text { Chi- } \\
\text { Square }\end{array}$ & $\mathrm{p}$-value \\
\hline Age & & & 58.1 & 0.000 \\
\hline$\leq 64$ & $92(8.39)$ & $1,108(16.28)$ & & \\
\hline $65-84$ & $363(33.12)$ & 2,391(35.13) & & \\
\hline$=>85$ & 641(58.49) & $3,308(48.6)$ & & \\
\hline Gender & & & 49.4 & 0.000 \\
\hline Male & $253(23.08)$ & $2,263(33.25)$ & & \\
\hline Female & $848(76.92)$ & $4,544(66.75)$ & & \\
\hline Heart & & & 11.3 & 0.001 \\
\hline Yes & $405(36.95)$ & $2,165(31.81)$ & & \\
\hline No & $691(63.05)$ & $4,642(68.19)$ & & \\
\hline \multicolumn{5}{|l|}{ Stroke } \\
\hline Yes & $119(10.86)$ & $748(10.99)$ & 0.0166 & 0.898 \\
\hline No & $977(89.14)$ & $6,059(89.01)$ & & \\
\hline \multicolumn{5}{|l|}{$\begin{array}{c}\text { Alzheimer"s/ } \\
\text { other Dementia }\end{array}$} \\
\hline Yes & $560(51.09)$ & $2,819(41.41)$ & & \\
\hline No & $536(48.91)$ & $3,988(58.59)$ & & \\
\hline COPD & & & 0.341 & 0.559 \\
\hline Yes & $113(10.31)$ & $742(10.90)$ & & \\
\hline No & $983(89.69)$ & $6,065(89.10)$ & & \\
\hline \multicolumn{5}{|l|}{$\begin{array}{c}\text { Paid by } \\
\text { medicaid }\end{array}$} \\
\hline Yes & $253(23.08)$ & $1,623(23.84)$ & 0.30 & 0.584 \\
\hline No & $843(76.92)$ & $5,184(76.16)$ & & \\
\hline
\end{tabular}

\section{Multivariable Logistic Regression Analyses}

Table 3 shows that all seven predictor variables are categorical variables. Age has two outputs since residents' age level $<65$ were used as a reference group. Binary logistic regression results showed that residents' age between 65-84 had 1.52 times odds of experiencing falls (or $52 \%$ increased odds) than the aged $\leq 64$ group with statistical significance ( $p$-value $<0.001$ ). Residents aged $\geq 85$ had 1.89 times odds of experiencing falls (or $89 \%$ increased odds) than residents who aged $\leq 64$ group with statistical significance ( $p$-value $<0.001$ ). We see that as age levels rise, the odds of falls also rises. Female residents had 1.47 times odds (or $47 \%$ increased odds) of experiencing falls than male residents with statistical significance ( $p$-value <0.001). Residents with heart disease had 1.19 times odds (or 19\% increased odds) of experiencing falls than residents without heart disease with statistical significance ( $p$-value $=0.013)$. Residents with stroke had 0.961 times odds of experiencing falls than residents without stroke. However, there were no statistically significant findings between falls and stroke ( $p$-value $=0.712)$. Residents with Alzheimer's disease/Dementia had 1.33 times odds of experiencing falls (or 33\% increased odds) 
than residents without Alzheimer's/Dementia ( $p$-value $<0.001$ ) with statistical significance. Residents with COPD had 0.951 times odds of experiencing falls than residents without COPD. However, there were no statistically significant findings between falls and COPD ( $p$-value $=0.647)$.

Table 3: Logistic Regression Predicting Likelihood of Falls.

\begin{tabular}{|c|c|c|}
\hline Variables & Falls OR(95\%CI)* & P-Value \\
\hline Age & & 0.001 \\
\hline$\leq 64$ & REF & \\
\hline $65-84$ & $1.521(1.17-1.96)^{*}$ & \\
\hline$=>85$ & $1.890(1.46-2.43)^{*}$ & \\
\hline Gender & & 0.000 \\
\hline Male & REF & \\
\hline Female & $1.466(1.25-1.70)^{*}$ & \\
\hline Heart & & 0.013 \\
\hline No & REF & \\
\hline Yes & $1.189(1.03-1.36)^{*}$ & \\
\hline Stroke & & 0.712 \\
\hline No & REF & \\
\hline Yes & $0.961(0.78-1.18)$ & \\
\hline $\begin{array}{l}\text { Alzheimer"s/other } \\
\text { Dementia }\end{array}$ & & 0.000 \\
\hline No & REF & \\
\hline Yes & $1.327(1.16-51)^{*}$ & \\
\hline COPD & & 0.647 \\
\hline No & REF & \\
\hline Yes & $0.951(0.76-1.17)$ & \\
\hline Paid by medicaid & & 0.037 \\
\hline No & REF & \\
\hline Yes & $1.186(1.01-1.39)^{*}$ & \\
\hline
\end{tabular}

Residents with Medicaid coverage had 1.19 times odds of experiencing falls (or 19\% increased odds) than residents without Medicaid coverage with statistical significance ( $p$-value $=0.037$ ). Overall, our model is statistically significant compared to an empty model. The multivariable logistic regression model was statistically significant $(\chi 2(8)=116.57, \mathrm{p}<0.001)$. Of the seven-predictor variables, only five variables were statistically significant: age, gender, heart disease, Alzheimer's /Dementia, and Medicaid status. COPD and stroke were a not-statistically significant predictor of falls.

\section{Discussion}

This study provides evidence from a nationally representative survey that residents' age, gender, heart disease, Alzheimer's/ Dementia, and Medicaid coverage status were significantly predicting the likelihood of falls among younger and older adults in residential care facilities. These results suggest that efforts by residential care facility administrators to reduce falls through careful monitoring of residents as well as to identify residents at higher risk of falls will improve the quality of life and prevent prolonged hospitalization [4]. Our results corroborate findings from other studies. According to Iinattiniemi et al study, the rate of fall injuries for adults 85 and older were almost four times higher than adults between 65 and 74 in the U.S. Our study findings mirror previous studies that examined the association between falls and gender. Women at or over the age of 70 years were reported to be $58 \%$ more likely than men to have a fall injury [9]. As aforementioned, age, gender, residents with heart disease, circulatory, or respiratory problems are risk factors that can increase hospitalizations and falls incidence among older adults [4].

The most interesting finding was that COPD was a notstatistically significant predictor of falls. COPD is a chronic health condition that can limit the patient's activity. It was hypothesized that older patients with a history of COPD could have an increased risk of falling compared to their healthy peers [14]. Although we hypothesized that stroke would be an essential predictor for falls, we did not find significant results. A possible explanation for this result is possibly due to only $11 \%$ of residents having reported having a stroke. Although these results differ from another published study, they found that among 170 individuals 80 adults with a mean age of 67.6 (who experienced a stroke and recently discharged home) are at higher risk of falling than individuals without stroke [15]. However, a possible explanation for this variance in our findings with this previously published study might be due to the location of their research, which took place at patients' homes. These findings have important implications for health policy to develop plans to prevent the incidence of falls among younger and older adults in residential care facilities. Residential care facilities and assisted living administrators should make every effort to reduce falls rate among younger and older adults. Considering the high rate of falls among adults in residential care facilities and the lack of evidencebased guidelines to prevent falls, additional guideline development studies on falls at residential care facilities are necessary [16]. Moreover, there is a need to investigate continually whether the available evidence-based guidelines are actually conveyed to practice and to assess its effectiveness.

Appropriate assessments immediately after admission, physical safety enhancements, and careful monitoring of residents who are at higher risk of incurring a fall should be implemented to prevent falls in residential care facilities [4]. There are several limitations to the study. First, our research group only analyzed the 2010 NSRCF data. Hence, a potential limitation is that this is older data. Second, our group utilized a cross-sectional study design where it is not possible to establish a temporal sequence (i.e., predictor variable occurred before the outcome variable) where we can make causal inference claims. Third, a lack of documentation due to relying on the facilities administrators could have led to underreporting 
in terms of rate of falls. Furthermore, the NSRCF information on resident health status and chronic health conditions is based on inperson interviews with the facility directors or designated staff and records kept at the facility but not of a doctor or clinician. This data collection method could lead to underestimating the prevalence of some health conditions, such as recall bias, where the interviewed person was not able to remember health conditions or falls correctly in the past 12 months [4]. Despite these limitations, this study improves our current knowledge and understanding of how age, gender, heart disease, Alzheimer's/Dementia, and Medicaid coverage status are predicting the likelihood of falls for assisted living residents. In future studies, more work will need to be done to determine the current (recent) risk factors that can increase the likelihood of falls in residential care facilities.

\section{Conclusion}

Our study examined the effects of stroke, heart disease, Alzheimer/dementia, COPD, age, gender, and Medicaid status on falls for younger and older adults in residential care facilities from the 2010 NSRCF data. Using nationally representative survey data, we found that resident's age, gender, heart disease, Alzheimer's/ Dementia, and Medicaid coverage status are significantly predicting the likelihood of falls in residential care facilities. However, COPD and Stroke were not a predictor of falls. Current findings add to a growing body of literature on factors or predictors that affect falls. Among older adults' falls are common. Falls have been associated with several different risk factors. Age or gender are risk factors that cannot be altered. However, many other fall risk factors can be controlled (e.g., muscle strength, number of medications, balance). Appropriate assessments can help to identify those residents who have an increased risk of falls. Assessment should include the underlying causes and plans to reduce the negative impact of falls in older adults.

\section{References}

1. Centers for Disease Control and Prevention. Web-based injury statistics query and reporting system. (WISQARS). National Center for Injury Prevention and Control, Centers for Disease Control and Prevention.

2. Ambrose A, Paul G, Hausdorff J (2013) Risk factors for falls among older adults: A review of the literature. Maturitas 75(1): 51-61.

\section{ISSN: 2574-1241}

DOI: $10.26717 /$ BJSTR.2020.30.005018

Jongwha Chang. Biomed J Sci \& Tech Res

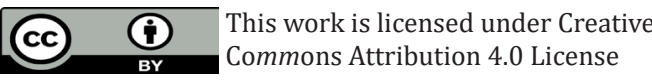

Submission Link: https://biomedres.us/submit-manuscript.php
3. Burns ER, Stevens JA, Lee R (2016) The direct costs of fatal and non-fatal falls among older adults -United States. Journal of Safety Research 58: 99-103.

4. Gimm G, Kitsantas P (2016) Falls Depression and Other Hospitalization Risk Factors for Adults in Residential Care Facilities. The International Journal of Aging and Human Development 83(1): 44-62.

5. Ruuskanen JM, Parkatti T (1994) Mobility and related factors among nursing home residents. Journal of the American Geriatrics Society 42(9): 987-991.

6. MacRae PG, Schnelle JF, Simmons SF, Ouslander JG (1996) Physical activity levels of ambulatory nursing home residents. Journal of Aging and Physical Activity 4(3): 264-278.

7. Jennifer C Macri, Andrea Iaboni, Julia G Kirkham, Colleen Maxwell, Sudeep S Gill, et al. (2017) Association between antidepressants and fallrelated injuries among long-term care residents. American Association for Geriatric Psychiatry 25(12): 1337-1338.

8. Sei Sato, Takeyasu Kakamu, Takehito Hayakawa, Tomohiro Kumagai, Tomoo Hidaka, et al. (2018) Predicting falls from behavioral and psychological symptoms of dementia in older people residing in facilities. Geriatrics Gerontology 18(11): 1573-1577.

9. Iinattiniemi S, Jokelainen J, Luukinen H (2019) Falls risk among a very old home-dwelling population. Scandinavian journal of primary health care 27(1): 25-30.

10. Dunlop D, Manheim L, Sohn M, Liu X, Chang R, et al. (2002) Incidence of functional limitation in older adults: The impact of gender, race, and chronic conditions. Archives of Physical Medicine And Rehabilitation 83(7): 964-971.

11. Deandrea S, Bravi F, Turati F, Lucenteforte E, La Vecchia C, et al. (2013) Risk factors for falls in older people in nursing homes and hospitals. A systematic review and meta-analysis. Archives of gerontology and geriatrics 56(3): 407-415.

12. Chang VC, Do MT (2015) "Risk factors for falls among seniors: implications of gender. American journal of epidemiology 181(7): 521531.

13. Dhargave P, Sendhilkumar R (2016) Prevalence of risk factors for falls among elderly people living in long-term care homes. Journal of clinical gerontology and geriatrics 7(3): 99-103.

14. Roig M, Eng J, Road J, Reid W (2009) Falls in patients with chronic obstructive pulmonary disease: A call for further research. Respiratory Medicine 103(9): 1257-1269.

15. Simpson L, Miller W, Eng J, Cifu D (2011) Effect of Stroke on Fall Rate, Location and Predictors: A Prospective Comparison of Older Adults with and without Stroke (Falls after Recent Stroke). PLoS ONE 6(4): e19431.

16. Jung D, Shin S, Kim H (2014) A fall prevention guideline for older adults living in long-term care facilities. International nursing review 61(4): 525-533.

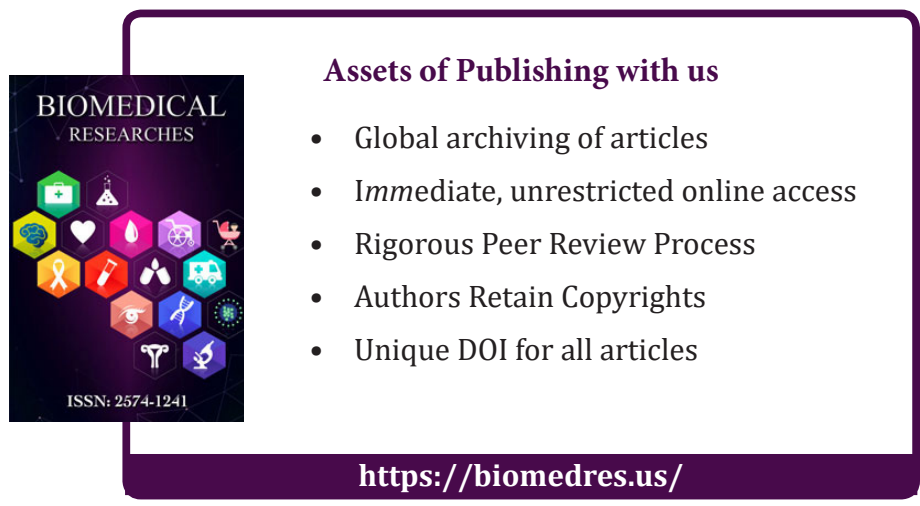

